\title{
BRIEF COMMUNICATION The association of aspirin use with severity of acute exacerbation of chronic obstructive pulmonary disease: a retrospective cohort study
}

\author{
Tadahiro Goto $\mathbb{D}^{1}$, Mohammad Kamal Faridi ${ }^{1}$, Carlos A. Camargo ${ }^{1}$ and Kohei Hasegawa ${ }^{1}$
}

Little is known about the effect of long-term aspirin use on acute severity of COPD. We hypothesized that, in patients hospitalized for acute exacerbation of COPD (AECOPD), long-term aspirin use is associated with lower risks of disease severity (in-hospital death, mechanical ventilation use, and hospital length-of-stay). We conducted a retrospective cohort study using large population-based data from 2012 through 2013. Among 206,686 patients (aged $\geq 40$ years) hospitalized for AECOPD, aspirin users had lower inhospital mortality (1.0 vs. $1.4 \%$; OR 0.60 [95\% Cl $0.50-0.72]$; $P<0.001)$ and lower risk of invasive mechanical ventilation use $(1.7$ vs. 2.6\%; OR 0.64 [95\% Cl 0.55-0.73]; $P<0.001$ ) compared to non-users, while there was no significant difference in risks of noninvasive positive pressure ventilation use. Length-of-stay was shorter in aspirin users compared to non-users $(P<0.001)$. In sum, in patients with AECOPD, aspirin use was associated with lower rates of in-hospital mortality and invasive mechanical ventilation use, and shorter length-of-stay.

npj Primary Care Respiratory Medicine (2018)28:7 ; doi:10.1038/s41533-018-0074-x

Increasing evidence suggests a potential benefit of antiplatelet therapy on chronic outcomes in patients with chronic obstructive pulmonary disease (COPD). ${ }^{1,2}$ For example, a recent analysis of an ongoing prospective cohort study revealed that antiplatelet therapy was associated with a lower 1-year mortality among individuals with COPD. ${ }^{1}$ However, little is known about the effect of aspirin use on acute outcomes (including mortality) in patients hospitalized for acute exacerbation of COPD (AECOPD).

To examine the association of aspirin use with severity of AECOPD, we conducted a retrospective cohort study using data from the State Inpatient Databases (SID) of seven US states (Arkansas, Florida, lowa, Nebraska, New York, Utah, and Washington) from 2012 and 2013. Additional details of the SID may be found elsewhere. ${ }^{3}$ The institutional review board of Massachusetts General Hospital approved this study.

We identified all hospitalizations made by patients aged $\geq 40$ years with a primary discharge diagnosis of COPD (ICD-9-CM codes: 491.21, 491.22, 491.8, 491.9, 492.8, 493.20, 493.21, 493.22, and 496), or those with a primary diagnosis of respiratory failure (codes: 518.81, 518.82, 518.84, and 799.1) and a secondary diagnosis of COPD. ${ }^{4}$ We included only the first hospitalization for AECOPD for each patient during the study period. Then, we further identified aspirin users by using the ICD-9-CM code for long-term (current) use of aspirin (V58.66) in any diagnostic field at the hospitalization. ${ }^{5}$

The outcome measures were in-hospital death (SID does not include the information on the cause of death), the use of mechanical ventilation (invasive mechanical ventilation [ICD-9-CM procedure code 96.04] and non-invasive positive pressure ventilation [NIPPV, code 96.30] assessed separately), and hospital length-of-stay (LOS). To examine the association between aspirin use and each outcome, we fit unadjusted and adjusted logistic regression or negative binomial regression models with generalized estimating equations to account for patient clustering within hospitals. We adjusted for age, sex, race/ethnicity, payer, median household income, 27 Elixhauser comorbidity measures (including heart failure, arrhythmias, and valvular diseases), hospitalization year, and hospital state. We repeated the analyses with (1) excluding patients who had any of the cardiovascular comorbidities that are commonly treated with aspirin (coronary artery diseases [codes, 410-414] and ischemic stroke [codes, 433-434]) and (2) excluding these potential aspirin users and patients with heart failure. All analyses were performed using STATA 14.1 (StataCorp, College Station, TX).

Overall, we identified 206,686 patients with hospitalization for AECOPD in the seven US states. Of these, 13,826 patients (7\%) were aspirin users. Aspirin users were older, and more likely to have Medicare comorbidities, compared to non-users (all $P<$ 0.001 ; Table 1). Aspirin users had a significantly lower in-hospital mortality compared to non-users ( 1.0 vs. $1.4 \%$; adjusted OR 0.60 [95\% Cl 0.50-0.72] $P<0.001$; Table 2). Additionally, aspirin users had a significantly lower rate of invasive mechanical ventilation use (1.7 vs. 2.6\%; adjusted OR 0.64 [95\% Cl 0.55-0.73] $P<0.001$ ), while there was no significant difference in the rate of NIPPV use (7.6 vs. 7.2\%; adjusted $\mathrm{OR} 1.05$ [95\% Cl 0.98-1.12] $P=0.20$ ). Hospital LOS was significantly shorter in aspirin users compared to non-users (median, 3 days vs. 4 days), corresponding to $-7 \%$ change in the adjusted model $(95 \% \mathrm{Cl}-5$ to $-9 \%, P<0.001)$. In the sensitivity analysis excluding the patients with history of coronary artery diseases, ischemic stroke, and/or heart failure, the point estimates of these associations did not change materially.

Consistent with our findings, previous cohort studies have reported that, among patients with COPD, the use of antiplatelet therapy is associated with a lower long-term mortality. ${ }^{6}$

${ }^{1}$ Department of Emergency Medicine, Massachusetts General Hospital, 125 Nashua Street, Suite 920, Boston, MA 02114, USA

Correspondence: Tadahiro Goto (tag695@mail.harvard.edu)

Received: 8 October 2017 Revised: 25 January 2018 Accepted: 26 January 2018

Published online: 21 February 2018 
Table 1. Characteristics of patients hospitalized for acute exacerbation of chronic pulmonary obstructive disease, according to aspirin use

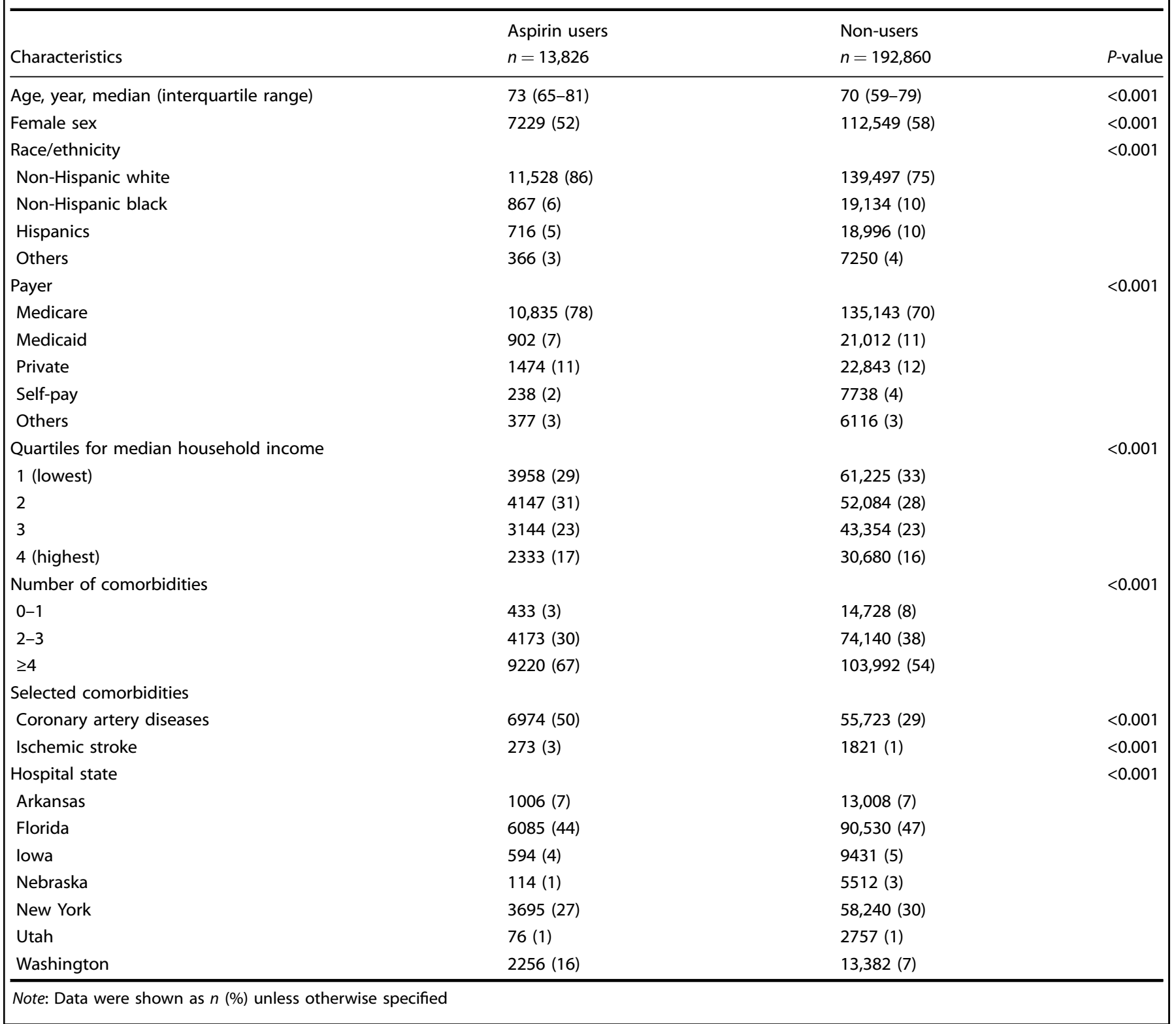

Additionally, an observational study of 1343 patients hospitalized for AECOPD in the UK reported that antiplatelet users (aspirin or clopidogrel) have a non-significantly lower odds of in-hospital mortality. ${ }^{1}$ Our population-based study from seven US states, with a much larger sample size, extends these findings by demonstrating the significant association of long-term aspirin use with lower acute severity and mortality of AECOPD.

The nature of aspirin-acute severity association in COPD warrants further investigation. The increased platelet activation in patients with $\mathrm{AECOPD}^{7}$ contributes to microvascular thrombosis that leads to organ ischemia and tissue damage. ${ }^{8}$ The use of aspirin may impede the inflammatory pathways secondary to the activated state of platelets and prevent microvascular thrombus formation by inhibiting the surface expression of adhesion molecules. ${ }^{8}$ These mechanisms for potential benefits of aspirin have also been indicated in other acute conditions accompanying systemic inflammation, such as sepsis and acute respiratory distress syndrome. ${ }^{9}$ Furthermore, the aspirin use potentially reduced the risk of cardiovascular diseases-related death, mechanical ventilation use, and longer LOS. This potential mechanism may be supported by the observed attenuated associations between aspirin use and severity of AECOPD in patients without cardiovascular diseases.

Our inferences may be limited by the low sensitivity of $I C D-9-C M$ code for long-term (current) aspirin use. ${ }^{6}$ However, the results were consistent in the sensitivity analysis excluding potential aspirin users that addressed the under-coding among patients who should have been treated with antiplatelets. Additionally, our inferences might not be generalizable to patients with less-thansevere AECOPD. Nevertheless, our data remain highly relevant for 700,000 patients hospitalized yearly in the US, ${ }^{10}$ a population with high morbidity.

In sum, by using a large population-based data set, we found that long-term aspirin users have lower rates of in-hospital mortality and invasive mechanical ventilation use, and shorter LOS. Although causal inferences remain premature, in conjunction with the prior studies, ${ }^{1,2,6}$ aspirin-a widely used and inexpensive medication-may be a potential therapeutic option for patients with COPD. 
Table 2. Unadjusted and adjusted associations between aspirin use and severity of acute exacerbation of chronic obstructive pulmonary disease

\begin{tabular}{|c|c|c|c|c|c|c|}
\hline Outcomes & Aspirin users & Non-users & $\begin{array}{l}\text { Unadjusted association } \\
(95 \% \mathrm{Cl})\end{array}$ & $P$-value & $\begin{array}{l}\text { Adjusted association } \\
(95 \% \mathrm{Cl})\end{array}$ & $P$-value \\
\hline \multicolumn{7}{|l|}{ Main analysis } \\
\hline Invasive mechanical ventilation ${ }^{a}$ & $1.7 \%(1.5-1.9 \%)$ & $2.6 \%(2.5-2.6 \%)$ & $0.64(0.56-0.74)$ & $<0.001$ & $0.64(0.55-0.73)$ & $<0.001$ \\
\hline NIPPV use $^{a}$ & $7.6 \%(7.2-8.1 \%)$ & $7.2 \%(7.1-7.3 \%)$ & $1.06(0.99-1.13)$ & 0.06 & $1.05(0.98-1.12)$ & 0.20 \\
\hline Hospital LOS, days, median (IQR) ${ }^{\mathrm{b}}$ & $3(2-5)$ & $4(2-6)$ & $-6 \%(-4$ to $-7 \%)$ & $<0.001$ & $-7 \%(-5$ to $-9 \%)$ & $<0.001$ \\
\hline In-hospital death ${ }^{\mathrm{a}}$ & $1.0 \%(0.8-1.3 \%)$ & $1.3 \%(1.1-1.3 \%)$ & $0.85(0.67-1.09)$ & 0.20 & $0.72(0.56-0.93)$ & 0.01 \\
\hline Invasive mechanical ventilation ${ }^{a}$ & $1.8 \%(1.5-2.1 \%)$ & $2.4 \%(2.3-2.5 \%)$ & $0.74(0.61-0.89)$ & 0.002 & $0.71(0.59-0.87)$ & 0.001 \\
\hline NIPPV use ${ }^{a}$ & $8.0 \%(7.3-8.7 \%)$ & $7.1 \%(7.0-7.2 \%)$ & $1.15(1.05-1.27)$ & 0.002 & $1.10(0.99-1.21)$ & 0.053 \\
\hline Hospital LOS, days, median (IQR) ${ }^{\mathrm{b}}$ & $3(2-5)$ & $3(2-5)$ & $-2 \%(0$ to $-5 \%)$ & 0.10 & $-5 \%(-2$ to $-8 \%)$ & $<0.001$ \\
\hline \multicolumn{7}{|l|}{ Sensitivity analysis $2^{d}$} \\
\hline \multicolumn{7}{|c|}{$\begin{array}{l}\text { Cl confidence interval, NIPPV non-invasive positive pressure ventilation, IQR interquartile range } \\
\text { aOdds ratio of aspirin use for each outcome in comparison with non-aspirin use, by using logistic regression model with generalized estimating equation to } \\
\text { account for patient clustering within hospitals } \\
\text { bPercent change in hospital LOS, by using negative binomial model with generalized estimating equation to account for patient clustering within hospitals } \\
{ }^{c} \text { Among patients without coronary artery diseases or ischemic stroke, } 6753 \text { patients were aspirin users and } 136,325 \text { patients were non-users } \\
{ }^{d} \text { Among patients without coronary artery diseases, ischemic stroke, or heart failure, } 5257 \text { patients were aspirin users and } 114,425 \text { patients were non-users }\end{array}$} \\
\hline
\end{tabular}

\section{DATA AVAILABILITY}

The State Inpatient Databases can be purchased from the website of Healthcare Cost and Utilization Project (https://www.hcup-us. ahrq.gov).

\section{ACKNOWLEDGEMENTS}

This study was supported by the grant R01 HS-023305 (Camargo) from the Agency for Healthcare Research and Quality (Rockville, MD).

\section{AUTHOR CONTRIBUTIONS}

T.G. takes responsibility for the paper as a whole. T.G., M.K.F., and K.H. conceived the study. C.A.C. obtained research funding. C.A.C. and K.H. supervised the conduct of the study. C.A.C. and K.H. provided statistical advice. T.G. and M.K.F. analyzed the data. T. G. drafted the manuscript, and all authors contributed substantially to its revision. The content is solely the responsibility of the authors and does not necessarily represent the official views of the Agency for Healthcare Research and Quality.

\section{ADDITIONAL INFORMATION}

Competing interests: The authors declare no competing interests.

Publisher's note: Springer Nature remains neutral with regard to jurisdictional claims in published maps and institutional affiliations.

\section{REFERENCES}

1. Harrison, M. T. et al. Thrombocytosis is associated with increased short and long term mortality after exacerbation of chronic obstructive pulmonary disease: a role for antiplatelet therapy? Thorax 69, 609-615 (2014).

2. Ekstrom, M. P., Hermansson, A. B. \& Strom, K. E. Effects of cardiovascular drugs on mortality in severe chronic obstructive pulmonary disease. Am. J. Respir. Crit. Care Med. 187, 715-720 (2013).

3. Overview of the State Inpatient Databases (SID). Healthcare cost and utilization project. Agency for healthcare research and quality, http://www.hcup-us.ahrq. gov/sidoverview.jsp (2017).
4. Yale New Haven Health Services Corporation/Center for Outcomes Research \& Evaluation. 2014 measures updates and specifications report hospital-level 30-day risk-standardized readmission measures, http://altarum.org/sites/ default/files/uploaded-publication-files/Rdmsn_Msr_Updts_HWR_0714_0.pdf (2014).

5. Bassett, J. C. et al. Gender, race, and variation in the evaluation of microscopic hematuria among Medicare beneficiaries. J. Gen. Intern. Med. 30, 440-447 (2015).

6. Pavasini, R. et al. Antiplatelet treatment reduces all-cause mortality in COPD patients: a systematic review and meta-analysis. Chron. Obstruct. Pulmon. Dis. 13, 509-514 (2016).

7. Maclay, J. D. et al. Increased platelet activation in patients with stable and acute exacerbation of COPD. Thorax 66, 769-774 (2011).

8. Gando, S. Microvascular thrombosis and multiple organ dysfunction syndrome. Crit. Care Med. 38, S35-S42 (2010).

9. Toner, P., McAuley, D.F. \& Shyamsundar, M. Aspirin as a potential treatment in sepsis or acute respiratory distress syndrome. Crit. Care 19, 374 (2015).

10. Ford, E. S. Hospital discharges, readmissions, and ED visits for COPD or bronchiectasis among US adults: findings from the nationwide inpatient sample 20012012 and Nationwide Emergency Department Sample 2006-2011. Chest 147, 989-998 (2015).

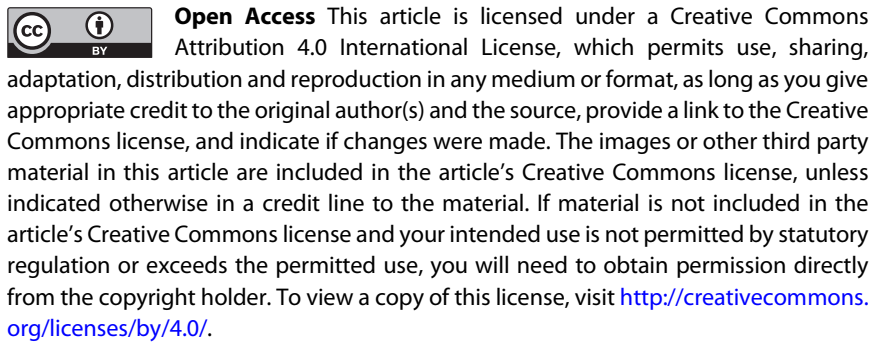

(c) The Author(s) 2018 